\title{
A comparison in university students of the amplitude of accommodation determined subjectively
}

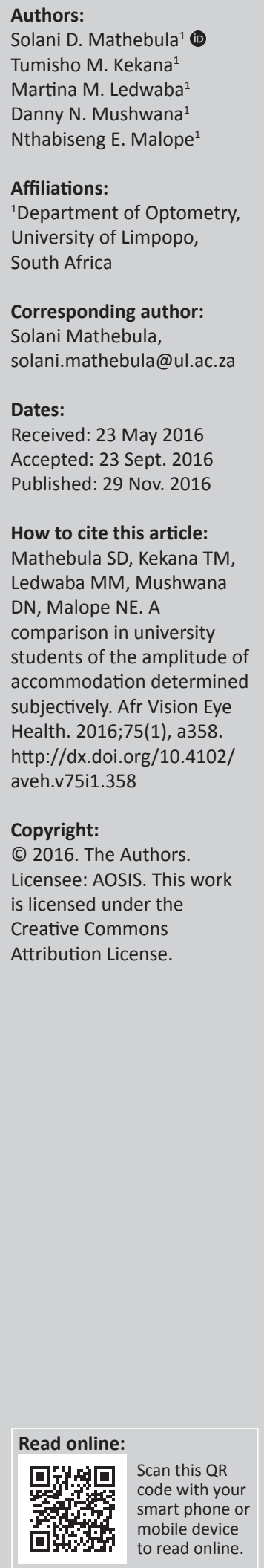

Background: Historically, the push-up and the minus lens methods have been used for the measurements of the amplitude of accommodation, and the differences between the results of these methods are well known.

Aim: The purpose of this study was to compare three methods for determining the monocular amplitude of accommodation and consider whether agreement exists between such methods.

Setting: The study was conducted at the Optometry Clinic, University of Limpopo.

Method: Thirty-four $(N=34)$ African optometry students participated in this study. There were 20 female and 14 male students. The age range of the participants was 20-34 years. Amplitude of accommodation was measured via the subjective push-up, push-down and minus lens methods only on the right eyes of the sample.

Results: The highest average amplitude of accommodation was obtained with the push-up method $(10.20 \mathrm{D} \pm 0.96 \mathrm{D})$, while the minus lens method produced the smallest mean amplitude of accommodation $(9.66 \mathrm{D} \pm 0.75 \mathrm{D})$. A higher correlation was found between the push-up and push-down methods $(r=0.80, p=0.06)$. The smallest correlation was observed between the push-up and the minus lens methods $(r=0.60, p=0.062)$. There were no statistically significant differences between the amplitude of accommodation in male and female students for all three methods $(p>0.005)$.

Conclusion: It seems easier to recognise the point where one can identify a target in pushdown amplitude than the point of first sustained blur in the push-up method. The push-up method tends to overestimate the actual amplitude of accommodation because of the effects of depth of focus. The less evaluated method in the literature is the push-away method; however, further research is necessary to answer the question of which (if any) method is more accurate.

\section{Introduction}

Accommodation can be defined as an increase in the refractive or dioptric power of the crystalline lens of the eye that enables the image of near objects of regard to be focused on the retina. ${ }^{1,2,3,4,5,6,7,8}$ Furthermore, accommodation can be defined as the eye's mechanism by which it adjusts its power to focus on objects at different distances. ${ }^{4}$ Changes in accommodation are mediated by parasympathetic innervation of the ciliary muscle of the eye from the Edinger-Westphal nucleus of the midbrain. ${ }^{1,2,3,4,5,6,7,8}$ According to the Helmholtz theory of accommodation, the ciliary muscle contracts in near vision. ${ }^{6}$ When the eye's power increases, the ciliary body is pulled forward, towards the immobile scleral spur. ${ }^{6,7}$ Simultaneously, the apex of the ciliary body is pulled towards the lens equator. This reduces the zonular tension at the lens equator, allowing the lens capsule to mould the lens into a more spherical and accommodated form. ${ }^{7}$ With accommodation, the lens diameter decreases, the lens equator moves away from the sclera and the curvature of the anterior and posterior lens surfaces changes, resulting in dioptric power increases in the lens and eye..$^{4,6,7}$ In distance vision, the crystalline lens is held in a relatively relaxed state under the tension of the zonules of Zinn (the suspensory ligament). ${ }^{1,2,3,4,5,6,7,8}$

Accommodation can also be defined as a change in the refractive or dioptric power of the eye that results from a change in the shape of the crystalline lens. ${ }^{8}$ The greatest increase in refractive power an eye can undergo is called the amplitude of accommodation or accommodative amplitude. Amplitude of accommodation declines progressively with age, but initially symptoms are masked by an increase in depth of focus and depth of field. ${ }^{1,2,3,4,5,6,7,8}$ An individual less than 40 years of age usually can see near objects with ease because their natural lens still has strong

Note: The article is based on data for the fourth-year undergraduate student research project under the supervision of the first author. (All students have since graduated with their Bachelor of Optometry degrees.) 
accommodative capabilities., ${ }^{90,11,12,13}$ Beyond age 40, presbyopia will eventually occur. Presbyopia is an age-related cause of visual impairment caused by the decline in accommodation that reduces the ability of the eye to focus on near objects. This process usually becomes noticeable between ages 40 and 50 but with ageing use of corrective lenses becomes necessary. It is theorised that stiffening of the crystalline lens is the primary mechanism for presbyopia. $^{9}$

As individuals develop presbyopia, they present clinically with difficulty in near-vision tasks. ${ }^{1,2,3,4}$ These problems manifest earliest in individuals with hyperopia and with emmetropia at about 40 years of age, when the accommodative reserve becomes insufficient to focus on near objects. . $2,3,4^{-1}$ However, the loss of the amplitude of accommodation begins early in life and progress to around 55 years of age, when little or no accommodative ability remains..$^{1,2,3,4}$

Clinically and experimentally, several subjective and objective methods for the measurement of amplitude of accommodation have been described, $1,2,3,4,8,9,10,11,12,13,14,15,16,17,18,19,20$ but subjective methods are more frequently used to measure the amplitude of accommodation. They include the push-up, the push-down (push-away) and, in pre-presbyopia, the minus lens methods. Positive lens methods are often used as presbyopia advances. The objective methods include modified dynamic retinoscopy methods and the use of an open-field autorefractor. ${ }^{1,2,3,4,5,6,7,8}$

The purpose of this study was to determine and compare the amplitude of accommodation using three subjective methods, namely push-up, push-down and the minus lens methods. It does seem that there are advantages and disadvantages for each method; hence, the study was designed to compare similarities and differences of the three procedures for determining monocular amplitude of accommodation in participants between 20 and 34 years of age.

\section{Methods}

\section{Subjects}

This was an analytic-descriptive study, and subjects were recruited randomly from a list of fourth-year optometry students. Thirty-four (20 females and 14 males) participants met the following inclusion criteria: visual acuity of 20/20 or better in each eye at both $6 \mathrm{~m}$ and $40 \mathrm{~cm}$ either with or without compensation, and no history of significant head or eye injuries, no ocular disease, not wearing contact lenses, no amblyopia and not currently taking any medications which might interfere with accommodation. The study included only right eyes from each of the 34 subjects. All subjects were made aware of the purpose of the study, and consent was obtained from each subject by means of a signed consent form. All investigations and measurements performed adhered to the tenets or principles, belief and requirements of the Declaration of Helsinki. All the measurements were performed by two researchers (T.M. and M.M.). Neither examiner knew the results of the other examiner.

\section{Procedure}

Refractive errors were determined initially using an autorefractor and later refined by subjective refraction using the phoropter. The monocular subjective refraction was followed by binocular balancing to a standard endpoint of maximum plus for best visual acuity. Three monocular methods were used to measure the amplitude of accommodation in the right eye of each subject. The tests were performed in the following order: (1) push-up, (2) push-down and (3) minus lens method. The push-up, push-down and the minus lens methods were performed by T.M.K. and M.M.L. For the push-up and push-down methods, the average distance from the subject was the near-point of accommodation. This value was converted to dioptres. Each examiner was unaware of the results obtained by the other examiner for each subject.

Each subject wore their distance correction determined from the result of subjective refraction, in which the most plus or least minus prescription for 20/20 visual acuity line was established. Each subject was asked to focus on a 20/20 row of letters of the near Snellen chart. Each test was performed monocularly on the right eye of each subject while the left eye was occluded. For the push-up and pushdown methods, the target was pushed towards or pulled away from the subject at a rate of approximately $5 \mathrm{~cm} / \mathrm{sec}$. All the measurements were carried out by the same researchers.

\section{Method 1: Push-up method}

Each subject was asked to focus on a 20/20 line at a distance of about $40 \mathrm{~cm}$, and the target was slowly moved towards the subject's fixating right eye along the ruler which was held near the visual acuity (VA) chart. As the target was slowly moving, the position of the overhead lamp was continuously adjusted to maintain approximately constant target luminance levels. Each subject was instructed to keep the letters as clear as possible and to report when there was the first slight sustained blurring of the letters. Subjects were repeatedly asked if the target was still clear as it was moved along the near-point ruler and reminded to report as soon as it becomes a little bit blurry. The endpoint was thus the first sustained blur, when the target could not be cleared after 3 seconds of viewing. ${ }^{1,2,3,4}$ The inverse of the final distance in metres (dioptres) from the target to spectacle plane was recorded as the subject's amplitude of accommodation.

\section{Method 2: Push-down or push-away}

The push-down method could be regarded as a variation of the push-up method. The accommodative target (20/20 line) was moved closer to the subject until blur was produced, and then the target was slowly pulled away from the subject until the subject could just read the 20/20 line of a Snellen chart. Again, the distance from the target to the spectacle plane was measured and converted to dioptres. The speed of the target movement was the same as the push-up method. 


\section{Method 3: Minus lens to blur}

With the right eye only, each subject viewed an illuminated 20/20 line of letters on the near Snellen chart, positioned at $40 \mathrm{~cm}$. With their distance correction in place, minus lens power was added in front of the right eye in $0.25 \mathrm{D}$ steps until the letters become and remained blurred that could not be cleared by further conscious effort. The minus lens amplitude of accommodation was taken as $2.50 \mathrm{D}$ (the dioptric equivalent of the working distance) plus the amount of minus lens power added (ignoring the sign). Some authors ${ }^{8,14}$ prefer to put the target at $33 \mathrm{~cm}$ rather than the established method of $40 \mathrm{~cm}$ as the minus lens method tends to make the target appear smaller and may make the patient more sensitive to identifying the first noticeable blur. However, placing the target closer to the subject may induce relative distance enlargement and may increase the possibility of overestimating amplitude of accommodation.

\section{Statistical analysis}

Data collected were analysed using SPSS version 23 software. Normality of the distribution of all samples was tested with the Kolmogorov-Smirnov test. The relationship between variables was tested using regression analysis. The means were also compared using the analysis of variance (ANOVA). A $p$-value of less than 0.05 was considered to have statistical significance.

\section{Results}

Amplitude of accommodation for the right eyes of 34 subjects was analysed. Of the 34 students who participated in the study, $20(58.8 \%)$ were women and $14(41.2 \%)$ men. The age range was $20-34$ years with a mean age of $24.62 \pm 3.70$ years. The mean age for female students was $24.9 \pm 3.7$ years, and $24.2 \pm 3.8$ years for male students. The independent-sample $t$-test was performed but did not show a significant difference between the mean ages of male and female students $(F=0.108$, $p=0.74)$. The descriptive statistics for the push-up, pushdown and minus lens methods are shown in Table 1. The Kolmogorov-Smirnov test showed that the amplitudes of accommodation were normally distributed $(p>0.05$ for all the procedures). Paired sample test was performed on all three procedures. The test showed a statistically significant

TABLE 1: Descriptive statistics for the amplitude of accommodation in dioptres with three different measuring methods.

\begin{tabular}{llll}
\hline Statistics & Push-up & Push-down & Minus lens \\
\hline Mean \pm s.d. & $10.93 \pm 0.95$ & $10.20 \pm 0.90$ & $9.66 \pm 0.75$ \\
Minimum & 8.75 & 8.75 & 8.00 \\
Maximum & 12.25 & 11.75 & 11.50 \\
95\% Cl for mean & Lower: 10.60 & Lower: 9.88 & Lower: 9.66 \\
& Upper: 11.25 & Upper: 10.20 & Upper: 10.20 \\
Median & 11.00 & 10.00 & 9.75 \\
Interquartile range & 1.50 & 1.50 & 1.00 \\
Skewness & -0.38 & 0.15 & 0.32 \\
Kurtosis & -0.61 & -0.93 & 0.57 \\
First quartile or $25 \%$ & 10.25 & 9.50 & 9.50 \\
Second quartile or $50 \%$ & 11.00 & 10.00 & 9.25 \\
Third quartile or $75 \%$ & 11.75 & 11.00 & 10.50 \\
\hline
\end{tabular}

$\mathrm{Cl}$, confidence interval; s.d., standard deviation. difference between the mean values obtained with the three methods $(p=0.00)$.

Figures 1 and 2 show the boxplots (or box-and-whisker plots) of the amplitudes of accommodation with the three methods. The horizontal bold line in the middle of the boxes represents

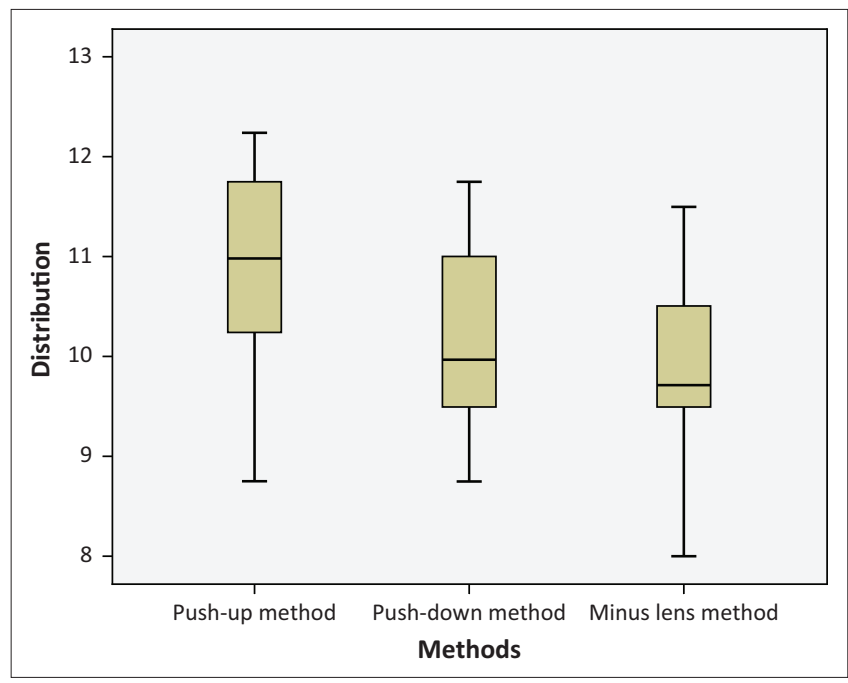

The edges of the box above and below the median are the quartiles (25th percent below and 75 th percent above). The box represents the middle most $50 \%$ of the distribution. The box has 'whiskers', one below the 1st quartile and one above the 3rd quartile. The bold horizontal line in the middle of the boxes represents the median (the 50th percentile) of each distribution. The whiskers indicate the lowest and highest values in each distribution. The highest median amplitude of accommodation was found with the push-up method.

FIGURE 1: Box plots of medians and interquartile ranges representing the measurements of the amplitude of accommodation for the three methods for all subjects. The $x$-axis represents the methods used to measure the amplitude of accommodation while the $y$-axis represents the distribution of the measurements of the amplitude of accommodation.

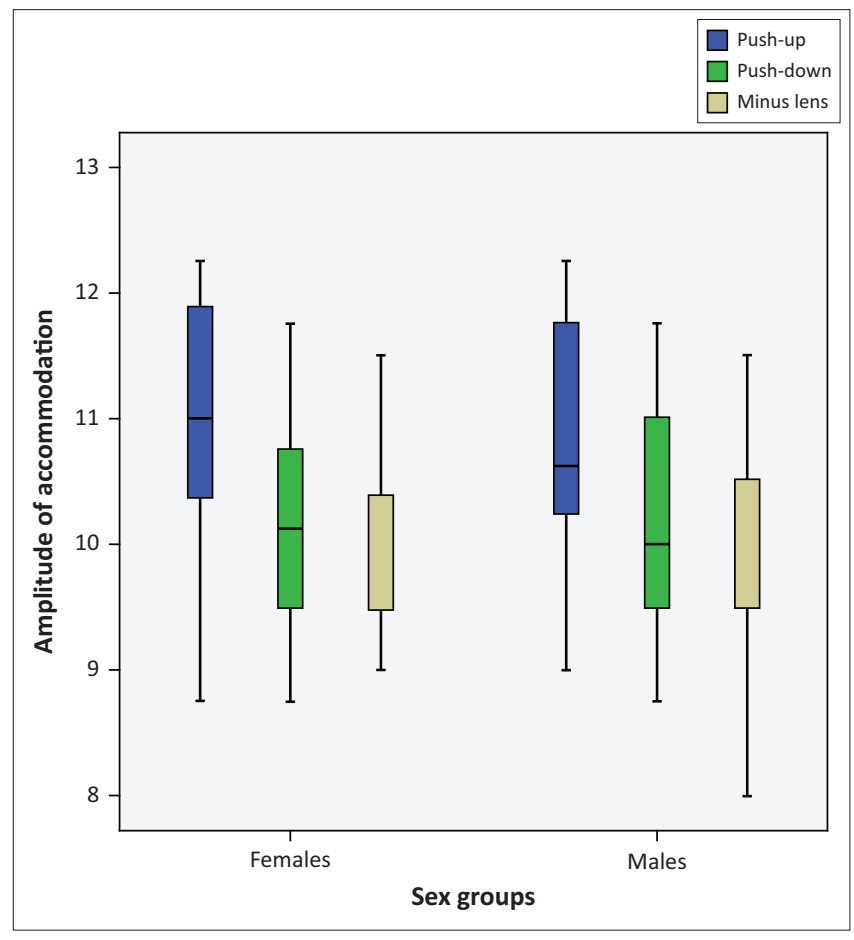

Higher amplitude of accommodation was recorded with the push-up method. Push-down and minus lens measurements showed a similar amplitude of accommodation.

FIGURE 2: Box plots for the amplitude of accommodation for the various methods of accommodation measurement for all subjects as a function of sex groups. The $x$-axis represents the methods used to measure the amplitude of accommodation while the $y$-axis represents the distribution of the measurements of the amplitude of accommodation. 
the median (50th percentile) of each distribution. The edges of the box above and below the median are the quartiles (25th percent below and 75th percent above). The box represents the middle most $50 \%$ of the distribution. The box has 'whiskers', one below the 1st quartile and one above the 3rd quartile. The whiskers indicate the lowest and highest values in each distribution. The boxplots showed that the distributions of the measurements are roughly symmetrical (more so for the push-up method in Figure 1). The measurements for each procedure are similarly dispersed in the boxplots; however, the measurements by the minus lens method were slightly skewed (Figure 1).

The relationships between variables were tested using the regression analysis (see Table 2 and Figures 3-6). Regression analysis is another way of analysing scatterplots. Table 2 shows the regression analysis performed on the amplitude of accommodation with the three methods. The correlation coefficient $(r)$ measures the strength of the relationship between two methods but not the agreement between. All the plots showed positive correlation (Figures 3-6). The closer the $r$ to 1 , the greater the strength of the linear relationship is. Also, the regression line $(y=a+b x)$ was used to predict the values of independent variables for a given dependent variable, where $y$ is the predicted response for any $x, a$ is the intercept of the line and $b$ is the slope of the line (Figures 3-6).

Correlation quantifies the degree to which two variables are related. However, a high correlation does not automatically imply that there is a good agreement between the two methods. The correlation coefficient and regression analysis

TABLE 2: Paired sample Pearson correlation coefficients for three procedures for amplitude of accommodation.

\begin{tabular}{llcc}
\hline Pairs & Methods & Correlation $(\boldsymbol{r})$ & Significance $(\boldsymbol{p})$ \\
\hline Pair 1 & Push-up and push-down & 0.80 & 0.056 \\
Pair 2 & Push-up and minus lens & 0.60 & 0.062 \\
Pair 3 & Push-down and minus lens & 0.67 & 0.015 \\
\hline
\end{tabular}

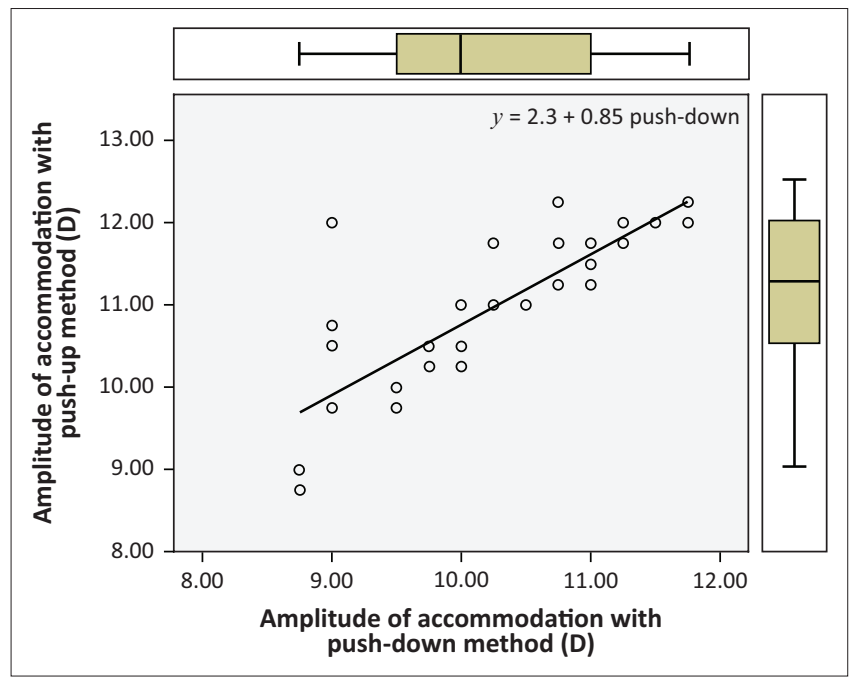

The linear regression line $r=0.80, p=0.056$. The measurements were highly correlated. The regression line is included.

FIGURE 3: Correlation plot of the amplitude of accommodation measured with the push-up method to first sustained blur and the push-down method to just clear.

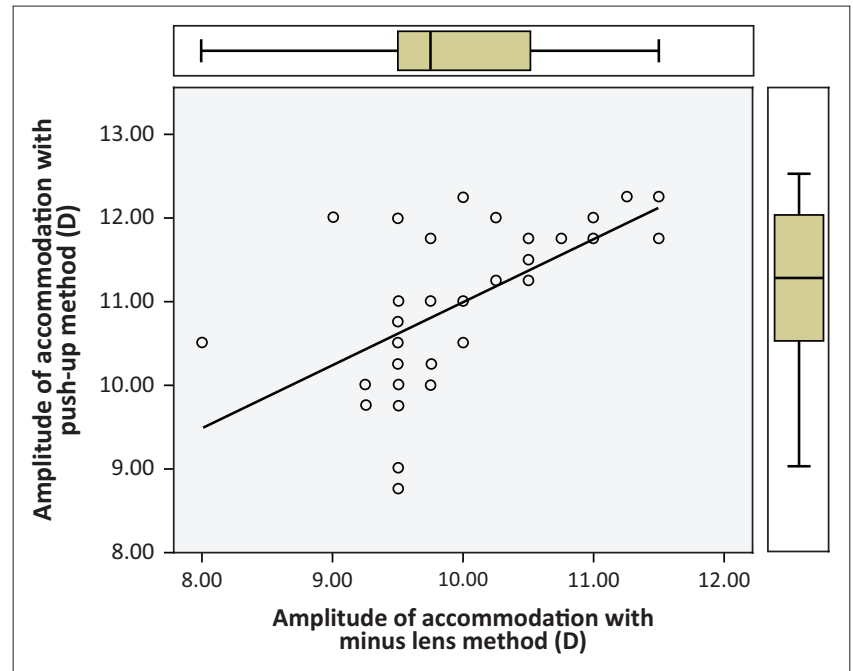

The correlation $r=0.60$. The regression line: $y=3.5+0.75 x$.

FIGURE 4: Correlation of the amplitude of accommodation measured using the push-up method versus the amplitude of accommodation measured using the minus lens method to blur.

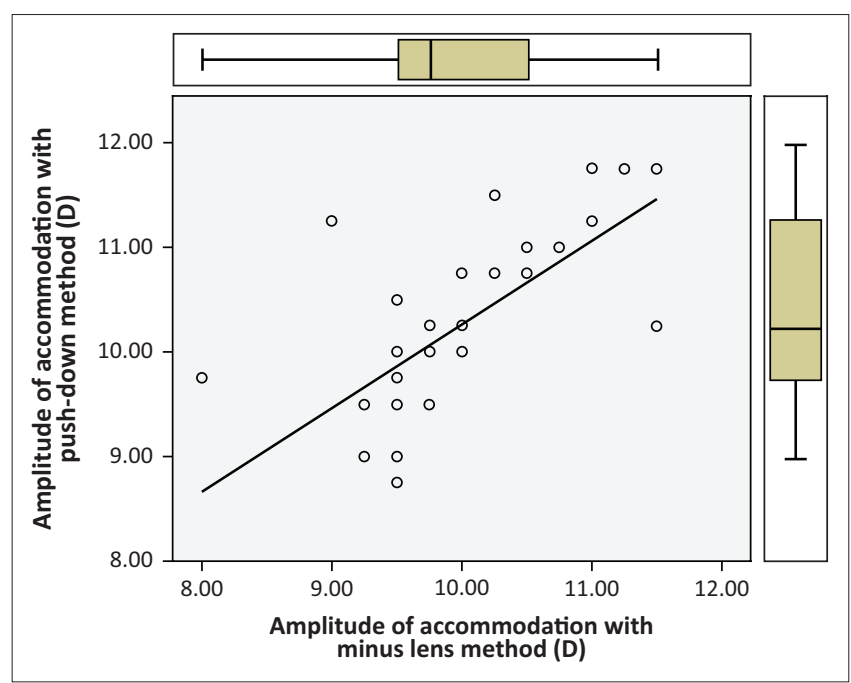

The least-square-fitted black line: $y=2.3+0.8$ (minus lens method). The correlation $r=0.67$.

FIGURE 5: Correlation between the amplitude of accommodation measured with the push-down method versus the amplitude of accommodation measured with the minus lens method.

are sometimes inadequate and can be misleading when assessing agreement, because they evaluate only the linear association of two sets of measurements. The Bland-Altman plot enables a visual inspection of the association between the differences in measurements and the magnitude for each pair of measurements of amplitudes of accommodation (Table 3 and Figure 6). The Bland-Altman shows the difference in measurements on the vertical axis against the average of the two measurements. Three horizontal lines are displayed on the plot. One horizontal line is drawn at the mean difference. The limits of agreement (LoAs) are indicated by the two broken lines on the plot, which are defined as the mean difference plus and minus 1.96 times the standard deviations of the differences. LoAs provide a way of assessing the range of variability between the two measurements. Bland-Altman plot is presented only for the push-up and push-down measurement methods. The same could be done for all other combinations of measurement methods. 


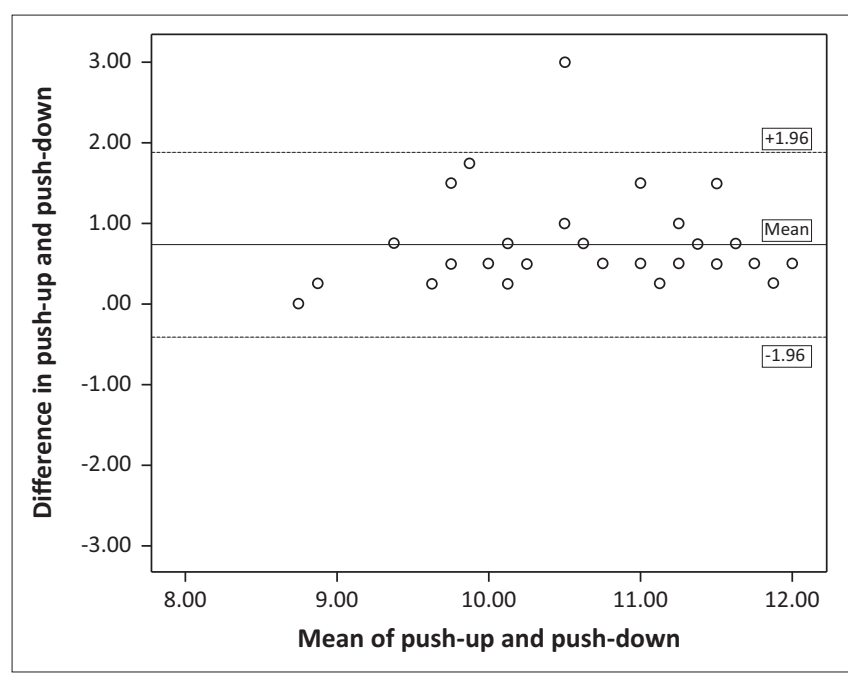

The solid horizontal line indicates the mean difference between the two measurements, and the horizontal dashed lines represent the $95 \%$ limit of agreement (mean \pm 1.96 s.d.). The $95 \%$ limits of agreement for the measurements are within 1.40 D.

FIGURE 6: A Bland-Altman plot of differences versus means for the push-up and push-down methods. The $y$-axis shows the difference between the two paired measurements (push-up - push-down) and the $x$-axis represents the mean of these measures [(push-up + push-down)/2].

TABLE 3: Mean differences between the values of the amplitudes of accommodation obtained using the three methods for measurement of amplitude of accommodation.

\begin{tabular}{llll}
\hline Statistics & $\begin{array}{l}\text { Push-up and } \\
\text { push-down }\end{array}$ & $\begin{array}{l}\text { Push-up and } \\
\text { minus lens }\end{array}$ & $\begin{array}{l}\text { Push-down and } \\
\text { minus lens }\end{array}$ \\
\hline Mean \pm SD & $0.74 \pm 0.59$ & $1.00 \pm 0.79$ & $0.27 \pm 0.68$ \\
Minimum & 0.00 & -0.75 & -1.25 \\
Maximum & 3.00 & 3.00 & 2.25 \\
$95 \% \mathrm{Cl}$ for the mean & 0.53 & 0.73 & 0.34 \\
& 0.94 & 1.28 & 0.51 \\
Interquartile range & 0.31 & 0.75 & 0.56 \\
Skewness & 2.10 & 0.50 & 0.53 \\
Kurtosis & 5.70 & 1.01 & 0.73 \\
\hline
\end{tabular}

$\mathrm{Cl}$, confidence interval.

\section{Discussion}

This study of 34 right eyes of young adults, aged 22-34 years, suggests that the push-up method had higher mean amplitude of accommodation as compared with the other two subjective methods. The minus lens method for the amplitude of accommodation showed the lowest average amplitude of accommodation of the three methods studied. Several studies ${ }^{11,13,14,15,16,17,18,19,20,21,22}$ also found the push-up method to show the highest amplitude of accommodation. The higher amplitude of accommodation by the push-up method could be as a result of the effect of the depth of focus that come into play during the push-up measurement. ${ }^{23}$ Also, Rosenfield and Gilmartin ${ }^{24}$ mentioned that the pushup method gives a higher value of the amplitude of accommodation because as the target distance decreases there is an increase in the angular size of the retinal image and also there is an increase in the proximal stimulation to the accommodation. Grosvenor ${ }^{3}$ reported that the depth of focus rather than the amplitude of accommodation is measured by the push-up method. Depth of focus is the extent to which the image may be positioned in front or behind the retina but still appear to be clear, ${ }^{24,25}$ whereas depth of field is the range of the object distances over which there is no detectable change in visual acuity or the visual acuity does not deteriorate. ${ }^{24,25,26}$ Previous studies have shown that generally, the push-up method overestimates the actual amplitude of accommodation because of factors including depth of focus, target size, illumination, proximal cues and endpoint criteria. ${ }^{16,24,25}$

In our study, there was no significant difference, however, between the push-up and push-down methods, even though the push-down method showed lower mean results for the amplitude of accommodation. Similarly, Anderson and Stuebing ${ }^{14}$ found no statistically significant difference between the push-up and push-down methods in a sample of 12 subjects. However, Koslowe et al. ${ }^{25}$ did find a statistically significant difference between these two methods. The lower results of the push-down method could be as a result of the psychophysical factors working in opposite directions for the two methods. ${ }^{14,25}$ It seems to be easier for people to recognise the point of identification (just readable) rather than the point of first sustained blur. The endpoint of first sustained blur can be a difficult concept for some patients to apply or the examiner may lead the patient to misinterpret the blur point by encouraging him or her to keep the target clear as long as possible. This could cause a delay in response that the target is blurred.

When determining the amplitude of accommodation using the minus lens method, there is also some minification of the retinal image because of the optical properties of the negative lenses. ${ }^{1,4}$ In the minus lens method, the relative distance magnification is absent and the proximal accommodation is constant. This method of minus lens should only be used under monocular conditions because it may result in an excess of accommodative convergence if performed binocularly and is likely to disrupt binocularity. The minus lens method gives lower results because it is performed in an unnatural environment where accommodation is being stimulated while the proximal cue is being reduced or denied. Rosenfield et al. ${ }^{27}$ mentioned that the push-up method overestimates the amplitude of accommodation measured with the minus lens method, while Rutstein et $a .^{28}$ reported that the push-up method overestimates minus lens amplitude of accommodation. Results of this study showed that the minus lens measurements of the amplitude of accommodation underestimated the amplitude of accommodation.

The difference between the push-up, push-down and minus lens methods could be as a result of proximally induced accommodative response as the target approaches the patient when performing the push-up method. Another explanation could be the target size. As the target approaches, the angle that it subtends increases, and this, in turn, delays the patient's ability to appreciate the end point (blur). With regard to the minus lens method, the minification of the target occurred as higher power lenses were introduced, leading to an underestimation of the true amplitude of accommodation. The underestimation of the amplitude of accommodation by the minus lens method was also seen even when the target is placed at $33 \mathrm{~cm} \cdot{ }^{29}$ 
However, comparing means alone might yield erroneous results as both outliers and sample size might affect the outcome. The coefficient of correlation measures the strength between two variables. It is possible that data obtained by two examiners using the same test conditions and procedures are not related. We used the Bland-Altman plot to determine the agreement between two methods and how close the data are to perfect agreement. The Bland-Altman plots showed that there is a good agreement between the push-up and push-down methods and between the push-down and minus lens methods, but the agreement was poor between the pushup and minus lens methods.

\section{Limitations of the study}

The study was limited only to normal young healthy subjects with good cooperation. All subjects were optometry students who were familiar with the procedures used. Symptomatic subjects were not included in the study. A much larger study is needed to verify our results.

Practitioner biases are another possible weakness of the study. Normally, the practitioner examining the patient expects or anticipates where the measurement endpoint should be and this may influence the result. The ideal situation to eliminate examiner bias is to use fully automated instruments, such as a retinoscope or wavefront aberrometer. Reaction time could be another limitation of the study. Reaction time may influence the push-up method detecting blur differently to the push-down method detecting clarity and may also influence the minus lens method if lenses are changed slow or fast. ${ }^{12}$

\section{Conclusion}

Subjectively measured amplitudes of accommodation may vary, even though they are routinely used clinically, they are not necessarily accurate measures of the amplitude of accommodation. The push-up method requires subjectively determined blur points, which may vary considerably both within and across subjects. It is possible that it is easier to recognise the point of identification in push-down than the point of first sustained blur in push-up. The push-up method is perhaps a less inappropriate method to measure the actual amplitude of accommodation because it overestimates the actual amplitude of accommodation. If the push-away method is to be used continually, it necessitates a larger study to determine such normative data.

\section{Acknowledgements}

The authors thank Dr Mologadi Ntsoane for her assistance in proofreading the manuscript.

\section{Competing interests}

The authors declare that they have no financial or personal relationships which may have inappropriately influenced them in writing this article.

\section{Authors' contributions}

S.D.M. conceived and designed the study and analysed the data, T.M.K. and M.M.L. performed the measurements and S.D.M., D.N.M. and N.E.M. wrote the article.

\section{References}

1. Rabbetts RB. Bennett \& Rabbetts' clinical visual optics. 4th ed. Oxford: ButterworthHeinemann; 2007.

2. Atchison DA, Copper EJ, McCabe KL. Critical subjective measurement of amplitude of accommodation. Optom Vis Sci. 1994;71(11):699-706. http://dx.doi.org/ 10.1097/00006324-199411000-00005

3. Grosvenor TP. Primary care optometry. 5th ed. Oxford: Butterworth-Heinmann; 2007.

4. Tunnacliffe AH. Introduction to visual optics. London, England: The Association of British Dispensing Opticians; 2001.

5. Hamasaki D, Ong J, Marg E. The amplitude of accommodation in presbyopia. Am J Optom Arch Am Acad Optom. 1956:33(1):3-14. http://dx.doi.org/10.1097/ 00006324-195601000-00002

6. Helmholtz H. Mechanism of accommodation. In: Southall JPC, editor. Helmholtz's treatise on physiological optics. New York: Dover; 1962.

7. Glasser A, Kaufman PL. The mechanism of accommodation in primates. Ophthalmology. 1999;106(5):863-872. http://dx.doi.org/10.1016/S0161-6420 (99)00502-3

8. Wold JE, Hu A, Chen S, Glasser A. Subjective and objective measurement of human accommodative amplitude. J Cataract Refract Surg. 2003;29(10):1878-1888. http:// dx.doi.org/10.1016/S0886-3350(03)00667-9

9. Glasser A, Campbell MCW. Presbyopia and the optical changes in the human crystalline lens with age. Vision Res. 1998;38(2):209-229. http://dx.doi.org/ 10.1016/S0042-6989(97)00102-8

10. Mordi JA, Ciuffreda KJ. Dynamic aspects of accommodation: Age and presbyopia. Vision Res. 2004;44(6):591-601. http://dx.doi.org/10.1016/j.visres.2003.07.014

11. Ang RET, Sarmiento JAS, Remo JTM, Martinez GHA, Canilao LMB. Measurement of accommodative amplitude using wavefront aberrometer. Philippine J Ophthalmol. 2015;40(1):3-10.

12. Momeni-Moghaddam H, Kundart J, Askarizadeh F. Comparing measurement techniques of accommodative amplitude. Indian J Ophthalmol. 2014;62(6): 683-687. http://dx.doi.org/10.4103/0301-4738.126990

13. Ostrin LA, Glasser A. Accommodation measurements in pre-presbyopic and presbyopic population. J Cataract Refract Surg. 2004;30(7):1435-1444. http:// dx.doi.org/10.1016/j.jcrs.2003.12.045

14. Anderson HA, Stuebing KK. Subjective versus objective accommodative amplitude: Preschool to presbyopia. Optom Vis Sci. 2014;91(11):1290-1301. http://dx.doi. org/10.1097/OPX.0000000000000402

15. Goss DA. Clinical accommodation testing. Curr Opin Ophthalmol. 1992;3(1):78-82. http://dx.doi.org/10.1097/00055735-199202000-00011

16. Rosenfield M, Cohen AS. Repeatability of clinical measurements of the amplitude of accommodation. Ophthalmic Physiol Opt. 1986;16(3):247-249. http://dx.doi. org/10.1016/0275-5408(95)00093-3

17. Kragha IK. Amplitude of accommodation: Population and methodological differences. Ophthalmic Physiol Opt. 1986;6(1):75-80. http://dx.doi.org/10.1111/ j.1475-1313.1986.tb00703.x

18. Leon AA, Medrano SM, Rosenfield M. A comparison of the reliability of dynamic retinoscopy and subjective measurements of amplitude of accommodation. Ophthalmic Physiol Opt. 2012;32(2):133-141. http://dx.doi.org/10.1111/j.1475 1313.2012.00891.x

19. Hokoda SC, Ciuffreda KJ. Measurement of accommodative amplitude in amblyopia. Ophthalmic Physiol Opt. 1982;2(3):205-212. http://dx.doi.org/10.1111/j.14751313.1982.tb00178.x

20. Chen AH, O'Leary DJ. Validity and repeatability of the modified push-up method for measuring the amplitude of accommodation. Clin Exp Optom. 1998;81(2): 63-71. http://dx.doi.org/10.1111/j.1444-0938.1998.tb06628.x

21. del Pilar Cacho M, Gatrcia-Munoz A, Garcia-Bernabeu JR, Lopez A. Comparison between MEM and Nott dynamic retinoscopy. Optom Vis Sci. 1999;76(9):650-655. http://dx.doi.org/10.1097/00006324-199909000-00023

22. Nemeth G, Tsorbatzoglou A, Vamosi P, et al. A comparison of accommodation amplitudes in pseudophakic eyes measured with three different methods. Eye. 2008;22(1):65-69. http://dx.doi.org/10.1038/sj.eye.6702519

23. Atchison DA, Charman WN, Woods RL. Subjective depth-of-focus of the eye. Optom Vis Sci. 1997;74(7):511-520. http://dx.doi.org/10.1097/00006324-19970700000019

24. Rosenfield M, Gilmartin B. Effect of target proximity on the open-loop accommodative response. Optom Vis Sci. 1990;67(2):74-79. http://dx.doi.org/ 10.1097/00006324-199002000-00002

25. Koslowe K, Glassman T, Tzanani-Levi C, Shnear E. Accommodative amplitude determination: Pull-away versus pull-up method. Optom Vis Dev. 2010;41(1): 28-32. 
26. Pollock J. Accommodation measurement - Clear or blur? Aust Orthopt J. 1989; 25:20-22.

27. Rosenfield M, Portello JK, Blustein GH, Jang C. Comparison of clinical techniques to assess the near accommodative response. Optom Vis Sci. 1996;73(6):382-388. http://dx.doi.org/10.1097/00006324-199606000-00005
28. Rutstein RP, Fuhr PD, Swiatocha J. Comparing the amplitude of accommodation determined objectivily and subjectively. Optom Vis Sci. 1993:70(6):496-500. http://dx.doi.org/10.1097/00006324-199306000-00008

29. Taub MB, Shallo-Hoffmann J. A comparison of three clinical tests of accommodation amplitude to Hofstetter's norm to guide diagnosis and treatment. Optom Vis Dev. 2012;43(4):180-190 